\title{
Propiedades psicométricas \\ del Instrumento de Evaluación de Funcionalidad en el Tratamiento para el Cáncer Cervicouterino (FACT-CX) en pacientes mexicanas
}

\section{Psychometric properties of the Functionality Assessment Instrument in Cervical Cancer Treatment (FACT-CX) in Mexican patients}

\author{
Óscar Galindo Vázquez ${ }^{1}$, Mayra Cu Menes ${ }^{2}$, David Isla Ortiz ${ }^{3}$, \\ Salim Abraham Barquet Muñoz ${ }^{4}$, Abel Lerma Talamantes ${ }^{5}$ \\ y Abelardo Meneses Garcia ${ }^{6}$
}

\begin{abstract}
Citación: Galindo Vázquez, O., Cu-Menes, M., Isla Ortiz, D., Barquet Muñoz, S.A., Lerma Talamantes, A, y Meneses García, A. (2021). Propiedades psicométricas del Instrumento de Evaluación de Funcionalidad en el Tratamiento para el Cáncer Cervicouterino (FACT-CX) en pacientes mexicanas. Psicología y Salud, 31(1), 155-164. https://doi.org/10.25009/pys.v31i1.2685.
\end{abstract}

\section{RESUMEN}

\begin{abstract}
Introducción: La calidad de vida en pacientes con cáncer cervicouterino es uno de los principales objetivos del tratamiento multidisciplinario, de modo que la evaluación válida y confiable de la misma reviste particular importancia. Objetivo: Determinar las propiedades psicométricas del Instrumento de Evaluación de Funcionalidad en el Tratamiento Para el Cáncer Cervicouterino (FACT-Cx) en pacientes mexicanas. Método: Participaron 200 pacientes con una edad promedio de 53 años. Se utilizó un diseño transversal no experimental. Instrumentos: FACT-CX, FACT-G, HADS y EORTCQLQ-C30. Análisis estadístico: Se realizó un modelo factorial exploratorio y confimatorio, así como de validez concurrente. Resultados: Se identificaron tres factores con 13 reactivos. La consistencia interna global fue aceptable y una varianza explicada de $56.72 \%$. El análisis factorial confirmatorio mostró indicadores adecuados, así como del ajuste de la estructura del modelo, lo que indica un patrón equilibrado y parsimonioso. La validez, medida por medio de la correlación con las medidas concurrentes, mostró resultados significativos. Discusión: El FACT-CX en pacientes con cáncer cervicouterino en población mexicana presentó características psicométricas adecuadas y robustas, tanto exploratorias como confirmatorias. La relevancia de los resultados obtenidos radica en que se trata de una población que puede requerir una detección oportuna mediante instrumentos válidos y confiables.
\end{abstract}

Palabras clave: Calidad de vida; Cáncer cervicouterino; Mujeres; México; Propiedades psicométricas.

\footnotetext{
${ }^{1}$ Servicio de Psicooncología, Instituto Nacional de Cancerología, Calle Av. San Fernando No.22, Sección XVI, 14080 Ciudad de México, México, tel. (55)56-28 04-00, exts. 60259 y 12059, correo electrónico: psigalindo@yahoo.com.mx. Artículo recibido el 5 de agosto de 2019 y aceptado el 6 de mayo de 2020.

${ }^{2}$ Servicio de Psicología Gineco-Oncológica, Instituto Nacional de Cancerología, Calle Av. San Fernando No.22, Sección Xvi, 14080 Ciudad de México, México, correo electrónico: mayra.qmenes@hotmail.com.

${ }^{3}$ Servicio de Ginecología-Oncológica, Instituto Nacional de Cancerología, Calle Av. San Fernando No.22, Sección Xvi, 14080 Ciudad de México, México, correo electrónico: islasurgery@hotmail.com.

${ }^{4}$ Servicio de Colposcopía, Instituto Nacional de Cancerología, Calle Av. San Fernando No.22, Sección Xvi, 14080 Ciudad de México, México, correo electrónico: salimbarquet@icloud.com.

${ }^{5}$ Instituto de Ciencias de la Salud (ICSa), Universidad Autónoma del Estado de Hidalgo, Carretera Pachuca-Actopan camino a Tilcuautla s/n, 42160 Pueblo San Juan Tilcuautla, Hgo., México, correo electrónico: aleta_44@yahoo.ca.

${ }^{6}$ Dirección General, Instituto Nacional de Cancerología, Calle Av. San Fernando No.22, Sección Xvi, 14080 Ciudad de México, México, correo electrónico: amenesesg@incan.edu.mx.
} 


\begin{abstract}
Introduction: Improving quality of life is one of the main objectives of multidisciplinary treatment in patients with cervical cancer, so their valid and reliable evaluation is a fundamental aspect. Objective: To determine the psychometric properties of the Functionality Assessment Instrument in Cancer Treatment-Cervical Cancer (FACT-CX) in Mexican patients. Method: A total of 200 female patients with an average age of 53 years participated. A non-experimental cross-sectional design was used. Instruments: FACT-CX, FACT-G, HADS and EORTC QLQ-C30. Statistical analysis: Exploratory and confirmatory factorial models and concurrent validity were performed. Results: Three factors were identified with 13 items. Overall internal consistency was 0.78 with an explained variance of $56.72 \%$. Confirmatory factor analysis showed adequate indicators and the adjustment of the structure of the model that indicate a balanced and parsimonious model and validity through correlation with concurrent measures, showed positive significant results. Discussion: FACT-CX presented adequate and robust exploratory and confirmatory psychometric characteristics in patients with cervical cancer in Mexican participants. These results are relevant because this is a population that may benefit from timely detection through valid and reliable instruments.
\end{abstract}

Keywords: Quality of life; Cervical cancer; Women; Mexico; Psychometric properties.

\section{INTRODUCCIÓN}

$\mathrm{E}$ 1 cáncer cervical es una enfermedad de las mujeres que viven en países en desarrollo, principalmente (Organización Mundial de la Salud [OMS], 2006), ocupa el cuarto lugar en el mundo en cuanto a incidencia y mortalidad (Bray et al., 2018), se halla entre los tres tipos de cáncer más comunes, siendo el más frecuente en 41 de 184 países; mientras que en América Latina es también el cáncer más común entre las mujeres (Bosch, 2016). En México ocupa el tercer lugar en incidencia, con un total de casi ocho mil casos anuales (World Health Organization [WHO], 2019). Por consiguiente, los desafíos que enfrentan los países en términos de acceso a la atención médica, recursos financieros y educación son factores que pueden influir en la calidad de vida de las mujeres que reciben tratamiento para esta enfermedad (Sabulei y Maree, 2019).
Analizar la afectación en la calidad de vida en pacientes con cáncer se ha vuelto un objetivo que exige a los profesionales de la salud evaluar los efectos de las intervenciones y tomar decisiones en conjunto con los pacientes. Teunissen et al. (2007), en una revisión sistemática de la literatura, identificaron 37 síntomas que pueden afectar la calidad de vida, y que se producen en más de $10 \%$ de los casos, y cinco síntomas (fatiga, dolor, falta de energía, debilidad y pérdida de apetito) se han observado en más de la mitad, lo que indica que la alta prevalencia de síntomas es una constante y que estos son el resultado de la enfermedad y de su tratamiento, los cuales pueden persistir varios años después de terminado el mismo. Lo anterior constituye una carga adicional para el paciente durante el restablecimiento de su salud.

Sabulei y Maree (2019) identifican que ciertas afectaciones en la calidad de vida, como insomnio e incontinencia urinaria, son problemas recurrentes incluso después del tratamiento, a lo que se suman frecuentemente las dificultades financieras, sobre todo durante la fase de tratamiento. Adicionalmente, síntomas como diarreas, polaquiuria, disuria, irritación y secreción vaginal abundante, irritación en la piel, cansancio y debilidad, resequedad, dispareunia, estrechamiento o acortamiento vaginal y alteraciones urinarias son asimismo frecuentes (Montes, Mullins y Urrutia, 2006)

En relación al área psicológica, Meyerowitz, Formenti, Ell y Leedham (2000) identificaron niveles elevados de depresión y estrés relacionados con un bajo nivel socioeconómico y la migración, hallando que el menor apoyo social y el estrés fueron predictores significativos de tales síntomas. Además, algunas variables, como los síntomas físicos asociados con la radiación, y barreras prácticas como el nivel educativo bajo o el difícil acceso a los servicios de salud, contribuyen significativamente en el tratamiento.

Las mujeres con cáncer cervicouterino presentan, pues, una mayor tasa de depresión, la cual se manifiesta con síntomas como preocupaciones y miedos en diversas áreas de su vida, como el apoyo social, estrés, sexualidad, síntomas físicos, infertilidad y pérdida de su feminidad (Montes et al., 2006). 
Incluso durante el periodo de sobrevida las afectaciones permanecen. Wenzel et al. (2005) reportaron que persisten ciertas secuelas predictoras de la calidad de vida, como el malestar emocional y espiritual, el afrontamiento desadaptativo y las preocupaciones sobre la infertilidad; de hecho, $59 \%$ de las pacientes expresaron que probablemente participarían en un programa de asesoramiento sobre los problemas psicosociales asociados al cáncer, y $69 \%$ señaló que habría asistido a un programa de grupo de apoyo durante el tratamiento inicial si se lo hubieran ofrecido.

Las pacientes con cáncer ginecológico requieren diversos programas de apoyo multidisciplinario durante y después del tratamiento. Estos programas deben establecerse tempranamente desde el momento del diagnóstico y continuarse a lo largo de la enfermedad y el tratamiento hasta el punto de la supervivencia, pues las necesidades de atención y apoyo no satisfechas pueden conducir a una pobre calidad de vida (Putri, Afiyanti, Ungsianik y Milanti, 2018).

Cella (1994) describió la calidad de vida mediante dos componentes fundamentales: la multidimensionalidad, que se refiere a la amplitud de las áreas que los pacientes consideran importantes para sus vidas, como su bienestar físico, funcional, emocional y social; la subjetividad, a su vez, alude al hecho de que la calidad de vida solamente puede entenderse a partir de la perspectiva del paciente. Kleiboer et al. (2010) mencionan que es necesario evaluar la calidad de vida de forma oportuna y concurrentemente al tratamiento para valorar íntegramente la efectividad del mismo, lo que se traduce en mejores resultados en beneficio del paciente, por lo que es necesario emplear instrumentos psicológicos válidos y confiables que, aplicados a pacientes ambulatorios y hospitalizados, permitan determinar los efectos y beneficios de los tratamientos.

Así, el Instrumento de Evaluación de Funcionalidad en Tratamiento para el Cáncer-Cáncer Cervicouterino (FACT-Cx en lo sucesivo), desarrollado por Monk, Huang, Cella, y Long (2005), es un instrumento válido y confiable para evaluar calidad de vida que ha demostrado su utilidad en diversos estudios clínicos y que ha sido validado en distintos países (Ding, Hu y Hallberg, 2012). En nuestro país no hay instrumentos validados o diseñados para evaluar la calidad de vida en pacientes con cáncer cervicouterino, por lo que el presente estudio tuvo como objetivo validar dicho instrumento en una muestra de pacientes mexicanas.

\section{MÉTODO}

\section{Participantes}

Una vez que el proyecto fue aprobado por el Comité de Investigación y Ética del Instituto Nacional de Cancerología (INCan), se invitó a participar a pacientes que asistían al servicio de consulta externa de la Unidad Funcional de Ginecología Oncológica de la referida institución. Los criterios de inclusión fueron tener un diagnóstico oncológico de cáncer cervicouterino en cualquier estadio; estar bajo tratamiento oncológico (de cualquier tipo o combinación) o en periodo de seguimiento, y saber leer y escribir. Se excluyó a pacientes con deficiencias cognitivas, auditivas o visuales severas que impidieran contestar el FACT-CX.

\section{Instrumentos}

\section{Cédula de identificación.}

Se elaboró una cédula de identificación de la participante en la cual se recabaron algunas variables sociodemográficas y clínicas, como edad, sexo, estado civil, escolaridad, tipo de diagnóstico, estadio de la enfermedad, en tratamiento actual o en periodo de seguimiento, peso, resultados de la ECOG (que es una forma práctica de medir la calidad de vida de la paciente, cuyas expectativas de vida cambian en el transcurso de meses, semanas e incluso días) y comorbilidad.

Instrumento de Evaluación de Funcionalidad en Tratamiento para el Cáncer Cervicouterino (FACT-CX) (Monk et al., 2005).

El FACT-Cx es un módulo específico, complementario al FACT-G (Cella et al., 1993), que evalúa calidad de vida en pacientes con cáncer en su versión general. En formato autoaplicable, contiene 15 reactivos, cada uno de los cuales se contesta en una escala Likert que va de 0 ("nada"), a 4 ("mucho"). Cada subescala produce un puntaje separado que se suma para obtener una puntuación total de la calidad de vida. 
Instrumento de Evaluación de Funcionalidad en Tratamiento Para el Cáncer-General (FACT-G) (Cella et al., 1993).

El FACT-G es una medida de calidad de vida en pacientes con cáncer en una versión general de 27 ítems. Consta de cuatro subescalas: Bienestar físico (PWB), Bienestar social (SWB), Bienestar emocional (EWB) y Bienestar funcional (FWB). Cuenta con propiedades psicométricas adecuadas, ha sido traducido a casi cincuenta idiomas y se utiliza ampliamente en todo el mundo (Brucker, Yost, Cashy, Webster y Cella, 2005).

\section{Escala Hospitalaria de Ansiedad y Depresión (HADS) (Zigmond y Snaith, 1983).}

La HADS es un instrumento autoaplicable conformado por 14 ítems que tiene como constructos síntomas de ansiedad y depresión. La subescala de Depresión está centrada en el concepto de anhedonia como síntoma nuclear de este cuadro clínico. La HADS está adaptada para población mexicana oncológica por Galindo et al. (2015), cuenta con una consistencia interna global de 0.86 y $48.04 \%$ de varianza explicada. La subescala de Ansiedad tiene una confiabilidad, medida con el coeficiente alfa de Cronbach, de 0.79 y la de Depresión de 0.80 .

Instrumento de Calidad de Vida de la Organización Europea para la Investigación y Tratamiento del Cáncer (EORTC-QLQ-C30)

(Aaronson et al. (2003).

El EORTC-QLQ-C30 es un instrumento evaluado en estudios multinacionales y multiculturales. Validado en población mexicana por Oñate et al. (2009), está diseñado como un instrumento autoadministrable que el paciente puede contestar en aproximadamente diez minutos. Evalúa cinco escalas funcionales: Física, De desempeño, Cognitiva, Emocional y Social, así como tres escalas sintomáticas: Fatiga, Dolor y Náusea/vómito. Es una escala global que mide la calidad de vida y otros síntomas que los pacientes oncológicos sufren frecuentemente.

\section{Análisis estadísticos}

Análisis exploratorio. Se obtuvieron los estadísticos de tendencia central de cada reactivo para conocer su distribución en las opciones de respuesta; después se determinaron los grupos extre- mos (cuartil 25 y 75), y en cada reactivo se llevó a cabo una prueba $t$ de Student para muestras independientes a fin de identificar la propiedad discriminativa de cada reactivo, eliminándose de los siguientes análisis aquellos que tuvieran una $p>0.05$. Se efectuaron a continuación análisis de tablas cruzadas para conocer la correlación entre ítems, estimándose el coeficiente alfa de Cronbach. Finalmente, a través de rho de Spearman se obtuvieron las correlaciones entre los factores del FACT-CX con los factores del FACT-G, la HADS y el EORTCQLQ-C30 para estimar la validez concurrente.

Análisis confirmatorio. Con los resultados exploratorios, se evaluó el ajuste del modelo de cuatro factores mediante un análisis factorial confirmatorio, usando para ello el método de máxima verosimilitud, que incluyó la identificación y especificación del modelo, la estimación de parámetros estandarizados (correlaciones $\mathrm{R}^{2}$, covarianzas, índices de modificación y proporciones críticas de las diferencias) y la evaluación del ajuste mediante la observación de límites aceptables de los estimadores, así como de no colinealidad en las variables medidas. Se estimaron los siguientes índices: $X^{2}$, razón $X^{2} / \mathrm{gl}$, índice de bondad de ajuste (GFI) y sus complementos (AGFI, TLI), así como el índice de bondad de ajuste comparativo (CFI) y la raíz cuadrada media del error de aproximación (RMSEA).

\section{Procedimiento}

Se solicitó inicialmente la autorización a la organización Facit.org para utilizar la versión en español para población mexicana. Esta traducción fue sometida a un jueceo por diez expertos en las áreas de oncología y psicooncología para evaluar validez de contenido, claridad, comprensión y pertinencia de reactivos, instrucciones y opciones de respuesta.

Mediante una prueba piloto realizada con 30 pacientes mexicanas se evaluó la versión derivada del proceso de jueceo del FACT-Cx, aplicándose un formato de registro con preguntas que tuvieron el propósito de evaluar si las pacientes comprendían los ítems, o si contenían expresiones confusas u ofensivas en los ítems, instrucciones y opciones de respuesta, de acuerdo a los lineamientos internacionales (Koller et al., 2007). Se registraron las observaciones, se revisaron sistemáticamente y se hicieron las modificaciones pertinentes. 


\section{RESULTADOS}

La muestra constó de 200 participantes con cáncer cervicouterino evaluadas en el Instituto Nacional de Cancerología (INCan) de la Ciudad de México. Las características generales descriptivas de la muestra se muestran en la Tabla 1.

Tabla 1. Descripción de la muestra en pacientes con cáncer cervicouterino $(\mathrm{N}=200)$. Los valores se muestran en frecuencias y porcentajes o en medianas (percentil 25-percentil 75).

\begin{tabular}{|c|c|}
\hline \multirow{2}{*}{ Edad (años) } & $x=53,(44-60)$ \\
\hline & $\mathbf{f}(\%)$ \\
\hline \multicolumn{2}{|l|}{ Escolaridad } \\
\hline Ninguna o básica & $94(47)$ \\
\hline De media a superior & $106(53)$ \\
\hline \multicolumn{2}{|l|}{ Estado civil } \\
\hline Sin pareja & $100(50)$ \\
\hline Con pareja & $100(50)$ \\
\hline \multicolumn{2}{|l|}{ Comorbilidad } \\
\hline Sí & $55(28)$ \\
\hline No & $145(72)$ \\
\hline \multicolumn{2}{|c|}{ Antecedentes familiares de cáncer } \\
\hline Sí & $66(33)$ \\
\hline No & $134(67)$ \\
\hline \multicolumn{2}{|l|}{ Etapa clínica } \\
\hline Con metástasis & $23(12)$ \\
\hline Sin metástasis & $177(88)$ \\
\hline \multicolumn{2}{|l|}{ Periodo de tratamiento } \\
\hline En tratamiento activo & $50(25)$ \\
\hline En seguimiento & $150(75)$ \\
\hline \multicolumn{2}{|l|}{ Tratamientos recibidos } \\
\hline Dos o más tratamientos & $157(78)$ \\
\hline Sin tratamiento o hasta uno & $43(22)$ \\
\hline \multicolumn{2}{|l|}{ Número de comorbilidades } \\
\hline Al menos una comorbilidad & $189(94)$ \\
\hline Ninguna comorbilidad & $11(6)$ \\
\hline
\end{tabular}

\section{Análisis factorial exploratorio}

La escala FACT-Cx obtuvo en la prueba de esfericidad de Bartlett una $p$ de .001 , un índice KMO de 0.77 y una consistencia interna global de 0.78 . El reactivo 8 no discriminó en la prueba $t$ de Student; el reactivo 13 correlacionó por arriba de 0.847 con el reactivo 14 , siendo que este tiene un mayor per- fil clínico, por lo que se eliminaron del análisis. El estudio factorial exploratorio mediante el método de componentes principales y rotación varimax arrojó un modelo de tres factores (con coeficientes alfa de $.67, .67$ y .62, respectivamente), que explicó $56.72 \%$ de la varianza. Dichos factores e indicadores se asemejan a las características que se han reportado en otros estudios (Tabla 2). 
Tabla 2. Factores e indicadores.

\begin{tabular}{|c|c|c|c|c|c|}
\hline $\begin{array}{l}\text { Alfa de Cronbach total }=0.78 \\
\text { Media total de la escala }=36.8 \pm 7.9 \\
\mathrm{KMO}=0.77 \\
\text { Varianza total }=56.72 \% \\
\text { Prueba de esfericidad de Bartlett }=532.8 \mathrm{gl}=78 \\
p=0.001\end{array}$ & $\begin{array}{l}\text { Factor } 1 \\
(\mathrm{n}=5)\end{array}$ & $\begin{array}{c}\text { Factor } 2 \\
(\mathrm{n}=4)\end{array}$ & $\begin{array}{c}\text { Factor } 3 \\
(n=4)\end{array}$ & $\begin{array}{c}M \\
\text { del ítem }\end{array}$ & $\begin{array}{l}\text { D.E. } \\
\text { del ítem }\end{array}$ \\
\hline \multicolumn{6}{|l|}{ Factor 1. Síntomas vaginales } \\
\hline OP5. Siento la vagina muy estrecha o pequeña. & 0.754 & 0.074 & 0.023 & 2.83 & 1.25 \\
\hline OP10. Me molesta el estreñimiento. & 0.708 & 0.013 & 0.194 & 2.76 & 1.40 \\
\hline OP12. Tengo dificultad para controlar la orina. & 0.622 & 0.112 & -0.022 & 3.11 & 1.17 \\
\hline OP3. Tengo miedo a tener relaciones sexuales. & 0.542 & 0.271 & 0.162 & 2.49 & 1.49 \\
\hline OP14. Siento molestias al orinar. & 0.455 & 0.439 & -0.033 & 3.44 & 0.97 \\
\hline \multicolumn{6}{|l|}{ Factor 2. Preocupaciones } \\
\hline OP6. Estoy preocupada por mi capacidad de tener hijos. & -0.158 & 0.715 & 0.007 & 3.80 & 0.66 \\
\hline OP2. Tengo olor vaginal que me molesta. & 0.418 & 0.669 & 0.096 & 3.45 & 0.99 \\
\hline $\begin{array}{l}\text { OP7. Tengo miedo de que el tratamiento pueda hacerle } \\
\text { daño a mi cuerpo. }\end{array}$ & 0.198 & 0.641 & 0.264 & 2.80 & 1.36 \\
\hline OP1. Me molesta el flujo o sangrado por la vagina. & 0.364 & 0.594 & 0.158 & 3.36 & 1.09 \\
\hline \multicolumn{6}{|l|}{ Factor 3. Bienestar } \\
\hline OP.4 Me siento físicamente atractiva. & 0.014 & 0.068 & 0.755 & 2.11 & 1.19 \\
\hline OP9. Me gusta mi apariencia personal. & -0.027 & 0.216 & 0.663 & 2.30 & 1.22 \\
\hline OP11. Tengo buen apetito. & 0.069 & 0.148 & 0.657 & 2.52 & 1.15 \\
\hline OP15. Puedo comer lo que me gusta. & 0.203 & -0.081 & 0.606 & 1.83 & 1.19 \\
\hline Valor alfa del factor & 0.677 & 0.677 & 0.626 & & \\
\hline Porcentaje de varianza explicada. & $18.3 \%$ & $16.1 \%$ & $15.2 \%$ & & \\
\hline Media & 14.64 & 13.40 & 8.77 & & \\
\hline Desviación estándar & 4.19 & 3.00 & 3.27 & & \\
\hline Varianza del factor & 17.56 & 9.02 & 10.66 & & \\
\hline Correlación intraclase del factor & 0.296 & 0.344 & 0.295 & & \\
\hline Valor inferior & 0.230 & 0.269 & 0.221 & & \\
\hline Valor superior & 0.368 & 0.423 & 0.375 & & \\
\hline Valor F & 3.1 & 3.1 & 2.7 & & \\
\hline Valor $p$ & $\leq 0.001$ & $\leq 0.001$ & $\leq 0.001$ & & \\
\hline
\end{tabular}

\section{Análisis factorial confirmatorio}

Como comentario general para la interpretación de los datos agregados en la base de la Figura1, el valor de $X^{2}$ ajustado a 56 grados de libertad fue mucho menor a 3 , con un valor $p$ superior a 0.05 , lo que confirma un adecuado ajuste absoluto del modelo a los datos observados.

Los índices CFI, TLI y AGFI (bondad de ajustes comparativos relativos) superan el valor ideal $(\geq 0.95)$ cada uno, indicando que es el mejor modelo contrastado contra el nulo. El índice PNFI, que tiende a $1(>0.5)$ confirma que es un modelo de medición eficiente.

El índice RMR (muy próximo a 0 y menor que 0.08 ) confirma que la diferencia entre la matriz de covarianza observada y la predicha es casi nula. Por lo tanto, la discrepancia entre el modelo propuesto y los datos medidos es muy baja.

El RMSEA (radical del error de aproximación, o raíz cuadrada media de la razón entre el parámetro no central ajustado por los grados de libertad) es próximo a $0 \mathrm{y}$ el intervalo superior menor a 0.08 , confirmando así que el error del modelo es 
casi nulo. Finalmente, el tamaño muestral $(\mathrm{n}=200)$ no supera el calculado por la prueba de Hoelter $(\mathrm{N}=254, p=0.01)$ e indica que se acepta la hipóte- sis de que con esa muestra es posible afirmar que el modelo es correcto (con la probabilidad de un error alfa de 0.01).

Figura 1. Modelo de AFC de tres factores de primer orden y un factor general de segundo orden para el FACT-CX.

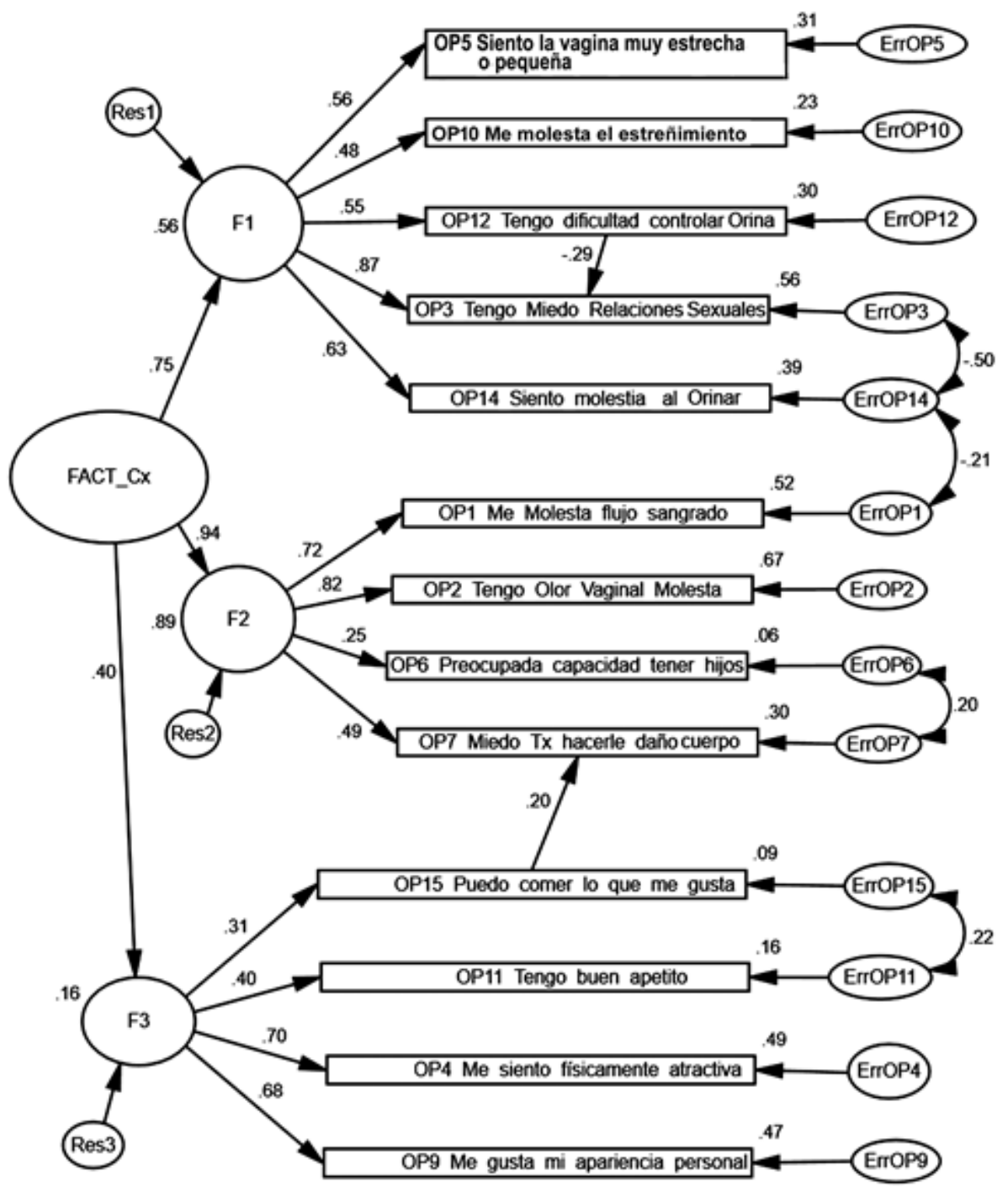

$X^{2}=65.57,56 \mathrm{gl}, X^{2} / \mathrm{gl}=1.171, p=0.179 ; \mathrm{CFI}=0.980, \mathrm{TLI}=0.972, \mathrm{AGFI}=0.925, \mathrm{RMR}=0.068, \mathrm{PNFI}=0.634, \mathrm{RMSEA}=0.029(0.000-0.055)$. Hoelter, $\mathrm{N}=254(p=0.01)$.

\section{Validez concurrente}

Las correlaciones entre las subescalas del FACT-CX y la escala funcional del EORTC QLQ 30 fueron positivas y estadísticamente significativas (rho de Spearman de 0.449 a $0.816, p<0.01$ ). Se identi- ficaron también las correlaciones inversas entre ese instrumento y las subescalas de síntomas del EORTC QLQ 30 (rho de Spearman de -0.300 a -0.539 ) y con la puntuación total y las subescalas de ansiedad y depresión del HADS ( $r$ de Spearman de .639 a $-.570, p<.05)$ (Tabla 3$)$. 
Tabla 3. Correlación de Spearman entre la escala FACT-Cx, FACIT-G, EORTC (QLQ30) y la HADS ( $\mathrm{n}=200$ ).

\begin{tabular}{|c|c|c|c|c|}
\hline & Puntaje total & Cx Factor 1 & Cx Factor 2 & Cx Factor 3 \\
\hline 1 FACIT G puntaje total & $.629(* *)$ & $.376(* *)$ & $.498(* *)$ & $.597(* *)$ \\
\hline FACIT-G Factor 1 & $.639(* *)$ & $.509(* *)$ & $.486(* *)$ & $.459(* *)$ \\
\hline FACIT-G Factor 2 & $.547(* *)$ & $.272(* *)$ & $.450(* *)$ & $.578(* *)$ \\
\hline FACIT-G Factor 3 & $.254(* *)$ & 0.056 & 0.138 & $.399(* *)$ \\
\hline FACIT-G Factor 4 & $.454(* *)$ & $.357(* *)$ & $.469(* *)$ & $.255(* *)$ \\
\hline \multicolumn{5}{|l|}{ EORTC QLQ30 } \\
\hline Salud Global Calidad de Vida & $-.504(* *)$ & $-.354(* *)$ & $-.362(* *)$ & $-.432(* *)$ \\
\hline \multicolumn{5}{|l|}{ Escalas funcionales } \\
\hline Función física & $.450(* *)$ & $.372(* *)$ & $.229(* *)$ & $.371(* *)$ \\
\hline Rol funcional & $.552(* *)$ & $.440(* *)$ & $.417(* *)$ & $.401(* *)$ \\
\hline Función emocional & $.562(* *)$ & $.458(* *)$ & $.354(* *)$ & $.397(* *)$ \\
\hline Función cognitiva & $.451(* *)$ & $.372(* *)$ & $.271(* *)$ & $.347(* *)$ \\
\hline Función social & $.568(* *)$ & $.412(* *)$ & $.486(* *)$ & $.417(* *)$ \\
\hline \multicolumn{5}{|l|}{ Escalas de Síntomas } \\
\hline Síntomas Fatiga & $.535(* *)$ & $.432(* *)$ & $.334(* *)$ & $.399(* *)$ \\
\hline Síntomas Náusea o vómito & $.439(* *)$ & $.306(* *)$ & $.401(* *)$ & $.323(* *)$ \\
\hline Síntomas Dolor & $.537(* *)$ & $.482(* *)$ & $.373(* *)$ & $.373(* *)$ \\
\hline \multicolumn{5}{|l|}{ Síntomas individuales } \\
\hline Disnea & $-.299(* *)$ & $-.249(* *)$ & $-.174(*)$ & $-.276(* *)$ \\
\hline Insomnio & $-.543(* *)$ & $-.457(* *)$ & $-.373(* *)$ & $-.346(* *)$ \\
\hline Pérdida de apetito & $-.570(* *)$ & $-.415(* *)$ & $-.389(* *)$ & $-.473(* *)$ \\
\hline Estreñimiento & $-.503(* *)$ & $-.554(* *)$ & $-.342(* *)$ & $-.224(* *)$ \\
\hline Diarrea & $-.314(* *)$ & $-.268(* *)$ & $-.181(*)$ & $-.211(* *)$ \\
\hline Dificultades financieras & $-.389(* *)$ & $-.348(* *)$ & $-.275(* *)$ & $-.271(* *)$ \\
\hline \multicolumn{5}{|l|}{ HADS } \\
\hline HADS Puntuación total & $-.585(* *)$ & $-.418(* *)$ & $-.412(* *)$ & $-.477(* *)$ \\
\hline HADS Ansiedad & $-.512(* *)$ & $-.433(* *)$ & $-.376(* *)$ & $-.345(* *)$ \\
\hline HADS Depresión & $-.551(* *)$ & $-.341(* *)$ & $-.374(* *)$ & $-.521(* *)$ \\
\hline
\end{tabular}

\section{DISCUSIÓN}

Pocos estudios se han realizado sobre calidad de vida en población mexicana con cáncer cervicouterino, por lo que poner mayor atención en la multidimensionalidad de la salud de este grupo de pacientes se ve como un aspecto fundamental aún pendiente por parte del equipo de salud. Lo anterior es relevante porque, de acuerdo a Fayers y Machin (2013), existe la necesidad de complementar los resultados oncológicos tradicionales con información sobre las opiniones de los pacientes, y cada vez se reconoce más que las mediciones de la calidad de vida cumplen este propósito. La me- dición desde la perspectiva de los pacientes puede guiar la gestión o ayudar a evaluar el tratamiento.

Sobre la adaptación del FACT-Cx a población mexicana, durante las entrevistas en el proceso de piloteo y aplicación, se identificó que, en términos generales, su comprensibilidad y administración tiene una factibilidad aceptable, lo que se ha corroborado previamente en la versión original (Ding et al., 2012) y en otras validaciones, lo que es esencial en una población con bajos niveles educativos y afectaciones en diferentes áreas de su vida durante o posteriormente a su tratamiento.

Una contribución del presente estudio es el análisis confirmatorio del FACT-Cx, lo cual no ha 
sido reportado de forma frecuente, en tanto que la versión mexicana ha demostrado una estructura teórica con tres factores de primer orden (síntomas físicos vaginales, preocupación y bienestar) y un factor general de segundo orden, con índices de bondad de ajuste adecuados y que se adapta apropiadamente a los datos empíricos de la muestra estudiada, que explican $56.72 \%$ de la varianza y una adecuada confiabilidad, lo que evidencia la calidad psicométrica de este instrumento. Respecto a la consistencia interna del FACT-Cx, es similar a la de la versión original y a la de estudios previos en otras poblaciones reportado por Tax (2017), con indicadores de entre .65 y .70 en las diferentes subescalas.

Los tres componentes identificados del FACT$\mathrm{Cx}$ de síntomas vaginales reflejan afectaciones frecuentes en el órgano afectado por el cáncer, preocupaciones asociados a los síntomas físicos y emocionales citados previamente en la literatura, así como un área de bienestar que refleja un nivel de funcionalidad adecuado en el aspecto físico y psicológico; sin embargo, es importante considerar la alta comorbilidad de $94 \%$ en el grupo de pacientes estudiadas, lo que implica la identificación de niveles de calidad de vida y síntomas de enfermedades frecuentes, como hipertensión arterial y diabetes, las cuales fueron las más prevalentes entre las participantes.

En relación a la validez concurrente, se identificaron valores de bajos a moderados estadísticamente significativos, principalmente con las variables y las subescalas del FACT-G y EORTC QLQ-30, lo que respalda las asociaciones que teórica y estadísticamente son las esperadas y que se han identificado en otros estudios. Las correlaciones con las subescalas de la HADS indican la estrecha relación entre los componentes del FACT-Cx y los aspectos psicológicos, lo que habrá de conducir a un trabajo más riguroso sobre la práctica clínica y la investigación de psicólogos y psiquiatras en pacientes con cáncer, e incluso más en la población latinoamericana, en la que las necesidades de salud mental del paciente oncológico siguen siendo una prioridad aún por resolver (Galindo y Costas, 2019).

El FACT-Cx tiene la ventaja de ser un instrumento fácil de administrar y confiable en la investigación y la práctica clínica. Las investigaciones futuras deberán evaluar si la detección de afectaciones mediante el FACT-CX en diferentes áreas de la calidad de vida aumenta las derivaciones al tratamiento oportuno multidisciplinario, y si estas consecuencias mejoran el cumplimiento y bienestar en estas pacientes. Se recomienda, pues, este instrumento para evaluar los efectos de intervenciones médicas y psicosociales que deriven en una adecuada calidad de vida y que se asocien con una apropiada adherencia terapéutica y funcionalidad. Su uso ofrece una guía al planear una evaluación exhaustiva y el diseño de tratamientos oncológicos.

En conclusión, el FACT-Cx para pacientes mexicanas con cáncer cervicouterino muestra características psicométricas adecuadas. La relevancia de lo anterior radica en poder detectar de forma oportuna a aquellas pacientes que podrían requerir atención multidisciplinaria en las diferentes áreas de su calidad de vida. La evaluación mediante instrumentos válidos y confiables de las necesidades reportadas por las pacientes debe ser una parte integral de las intervenciones en oncología, más aún en grupos con adversidad psicosocial que pueden ver su calidad de vida más afectada. Estas escalas deben estar relacionadas con la toma de decisiones sobre los tratamientos en conjunto con los equipos de salud multidisciplinarios, por lo que la calidad de vida deberá ser un aspecto central. Una limitación de este trabajo es que no se identificó la estabilidad a lo largo del tiempo del FACT-CX en pacientes que se encuentran en tratamiento activo.

\section{REFERENCIAS}

Aaronson, N.K., Ahmedzai, S., Bergman, B., Bullinger, M., Cull, A., Duez, N.J. et al. (1993). The European Organization for Research and Treatment of Cancer QLQ-C30: A quality-of-life instrument for use in international clinical trials in oncology. Journal of the National Cancer Institute, 85(5), 365-376.

Bosch, F.X. (2016). Eradication of cervical cancer in Latin America. Salud Publica de México, 58(2), 97-100.

Bray, F., Ferlay, J., Soerjomataram, I., Siegel, R.L., Torre, L.A. y Jemal, A. (2018). Global cancer statistics 2018: GLOBOCAN estimates of incidence and mortality worldwide for 36 cancers in 185 countries. CA: A Cancer Journal for Clinicians, 68(6), 394-424. 
Brucker, P.S., Yost, K., Cashy, J., Webster, K. y Cella, D. (2005). General population and cancer patient norms for the Functional Assessment of Cancer Therapy-General (FACT-G). Journal of Educational Evaluation for Health Professions, 28(2), 192-211.

Cella, D.F. (1994). Quality of life: concepts and definition. Journal of Pain Symptoms Manage, 9(3), $186-192$.

Cella, D.F., Tulsky, D.S., Gray, G., Sarafian, B., Linn, E., Bonomi, A. et al. (1993). The Functional Assessment of Cancer Therapy scale: development and validation of the general measure. Journal of Clinical Oncology, 11(3), 570-579.

Ding, Y., Hu, Y. y Hallberg, I.R. (2012). Psychometric properties of the Chinese version of the Functional Assessment of Cancer Therapy-Cervix (FACT-CX) for measuring health-related quality of life. Health Quality of Life Outcomes, $10(1), 124$.

Fayers, P.M. y Machin, D. (2013). Quality of life. The assessment, analysis, and interpretation of patient-reported outcomes (2nd ed.). Tronheim (Norway): Wiley.

Galindo V., O., Benjet, C., Juárez G., F., Rojas C., E., Riveros R., A., Aguilar P., J.L. y Alvarado A., S. (2015). Propiedades psicométricas de la Escala Hospitalaria de Ansiedad y Depresión (HADS) en una población de pacientes oncológicos mexicanos. Salud Mental, 38(4), 253-258. Doi: 10.17711/SM.0185-3325.2015.035.

Galindo V., O. y Costas M., R. (2019). Evidence-based psycho-oncology: A priority in mental health. Salud Mental, 42(3), 101-102. Doi: 10.17711/SM.0185-3325.2019.013.

Kleiboer, A., Gowing, K., Holm, H.C., Hibberd, C., Hodges, L., Walker, J. et al. (2010). Monitoring symptoms at home: what methods would cancer patients be comfortable using? Quality of Life Research, 19(7), 965-968.

Koller, M., Aaronson, N.K., Blazeby, J., Bottomley, A., Dewolf, L., Fayers, P. et al. (2007). Translation procedures for standardised quality of life questionnaires: The European Organization for Research and Treatment of Cancer (EORTC) approach. European Journal of Cancer, 43(12), 1810-1820.

Meyerowitz, B., Formenti, S., Ell, K. y Leedham, B. (2000). Depression among Latin cervical cancer patients. Journal of Social and Clinical Psychology, 19, 352-371. Doi: 10.1521/jscp.2000.19.3.352.

Monk, B.J., Huang, H.Q., Cella, D. y Long, H.J. III (2005). Quality of life outcomes from a randomized phase III trial of cisplatin with or without Topotecan in advanced carcinoma of the cervix: a Gynecologic Oncology Group Study. Journal of Clinical Oncology, 23(21), 4617-4625.

Oñate O., L.F., Alcántara P., A., Vilar C., D., García H., G., Rojas C., E., Alvarado A., S. et al. (2009). Validation of the Mexican Spanish version of the EORTC C30 and STO22 questionnaires for the evaluation of health-related quality of life in patients with gastric cancer. Annals of Surgical Oncology, 16(1), 88-95.

Sabulei, C. y Maree, J.E. (2019). An exploration into the quality of life of women treated for cervical cancer. Curationis, 42(1), e1-e9.

Teunissen, S.C., Wesker, W., Kruitwagen, C., de Haes, H.C., Voest, E.E. y de G., A. (2007). Symptom prevalence in patients with incurable cancer: a systematic review. Journal of Pain Symptoms Manage, 34(1), 94-104.

Wenzel, L., De Alba, I., Habbal, R., Kluhsman, B.C., Fairclough, D., Krebs, L.U. et al. (2005). Quality of life in long-term cervical cancer survivors. Gynecologic Oncology, 97(2), 310-317.

World Health Organization (2019). México: Globocan 2018. International Agency for Research on Cancer. Geneve: WHO.

Zigmond, A.S. y Snaith, R.P. (1983). The Hospital Anxiety and Depression Scale. Acta Psychiatrica Scandinavica, 67(6), 361-370. 